\title{
Longitudinal changes of ADHD symptoms in association with white matter microstructure: a tract-specific fixel-based analysis
}

\author{
Christienne G. Damatac ${ }^{a, b}$, Sourena Soheili-Nezhad ${ }^{a, b}$, Guilherme Blazquez Freches ${ }^{a, b}$, \\ Marcel P. Zwiers ${ }^{a, b}$, Sanne de Bruijn ${ }^{a, b}$, Seyma Ikde ${ }^{a, b}$, Christel M. Portengen ${ }^{a, b}$, \\ Amy C. Abelmann ${ }^{a, b}$, Janneke T. Dammers ${ }^{a, b}$, Daan van Rooij ${ }^{a, b}$, Sophie E. A. Akkermans ${ }^{a, b}$, \\ Jilly Naaijen ${ }^{\mathrm{a}, \mathrm{b}}$, Barbara Franke ${ }^{\mathrm{c}, \mathrm{d}}$, Jan K. Buitelaar ${ }^{\mathrm{a}, \mathrm{b}, \mathrm{e}}$, Christian F. Beckmann ${ }^{\mathrm{a}, \mathrm{b}, \mathrm{f}}$, \\ Emma Sprooten ${ }^{\mathrm{a}, \mathrm{b}}$
}

${ }^{a}$ Centre for Cognitive Neuroimaging, Donders Institute for Brain, Cognition and Behaviour, Radboud University, Nijmegen, The Netherlands

${ }^{b}$ Department of Cognitive Neuroscience, Donders Institute for Brain, Cognition and Behaviour, Radboud University Medical Center, Nijmegen, The Netherlands

${ }^{c}$ Department of Human Genetics, Donders Institute for Brain, Cognition and Behaviour, Radboud University Medical Center, Nijmegen, The Netherlands

d Department of Psychiatry, Donders Institute for Brain, Cognition and Behaviour, Radboud University Medical Center, Nijmegen, The Netherlands

e Karakter Child and Adolescent Psychiatry University Centre, Nijmegen, The Netherlands

${ }^{f}$ Centre for Functional MRI of the Brain, Wellcome Centre for Integrative Neuroimaging, Nuffield Department of Clinical Neurosciences, University of Oxford, John Radcliffe Hospital, Oxford, UK

Correspondence to Christienne G. Damatac, Department of Cognitive Neuroscience, Donders Centre for Cognition, Donders Institute for Brain, Cognition and Behaviour, Kapittelweg 29, 6525 EN Nijmegen, The Netherlands; email at christienne.damatac@donders.ru.nl. 
1

2

3

4

5

6

7

8

\section{Abstract}

Background: Variation in the longitudinal course of childhood attention deficit/hyperactivity disorder (ADHD) coincides with neurodevelopmental maturation of brain structure and function. Prior work has attempted to determine how alterations in white matter (WM) relate to changes in symptom severity, but much of that work has been done in smaller cross-sectional samples using voxel-based analyses. Using standard diffusion-weighted imaging (DWI) methods, we previously showed WM alterations were associated with ADHD symptom remission over time in a longitudinal sample of probands, siblings, and unaffected individuals. Here, we extend this work by further assessing the nature of these changes in WM microstructure by including an additional follow-up measurement (aged 18 - 34 years), and using the more physiologically informative fixel-based analysis (FBA). Methods: Data were obtained from 139 participants over 3 clinical and 2 follow-up DWI waves, and analyzed using FBA in regions-of-interest based on prior findings. We replicated previously reported significant models and extended them by adding another time-point, testing whether changes in combined ADHD and hyperactivity-impulsivity $(\mathrm{HI})$ continuous symptom scores are associated with fixel metrics at follow-up. Results: Clinical improvement in $\mathrm{HI}$ symptoms over time was associated with more fiber density at follow-up in the left corticospinal tract (ICST) ( $t_{\max }=1.092$, standardized effect[SE] $\left.=0.044, p_{\mathrm{FWE}}=0.016\right)$. Improvement in combined ADHD symptoms over time was associated with more fiber cross-section at follow-up in the ICST ( $\left.\mathrm{t}_{\max }=3.775, \mathrm{SE}=0.051, p_{\mathrm{FWE}}=0.019\right)$. Conclusions: Aberrant white matter development involves both ICST micro- and macrostructural alterations, and its path may be moderated by preceding symptom trajectory.

Keywords: attention deficit hyperactivity disorder; magnetic resonance imaging; diffusion imaging; white matter; microstructure

Abbreviations: ADHD: attention-deficit hyperactivity disorder; DTI: diffusion tensor imaging; DWI: diffusion-weighted imaging; FA: fractional anisotropy; FBA: fixel-based analysis; FC: fiber crosssection; FD: fiber density; FDC: fiber density and cross-section; FOD: fiber orientation distribution; $\mathbf{H I}$ : hyperactivity-impulsivity; IA: inattention; ICST: left corticospinal tract; ISLF: left superior longitudinal fasciculus; MRI: magnetic resonance imaging; SE: standardized effect; WM: white matter 


\section{Introduction}

Although (proto)typically considered a childhood syndrome, clinical trajectories of attentiondeficit/hyperactivity disorder (ADHD) vary by individual. Many ADHD-affected adolescents exhibit improvement over time, but approximately two-thirds of them retain impairing symptoms into adulthood [1-3]. The neural substrates that determine this variable clinical course of childhood ADHD have been increasingly investigated through the years, yet the dynamic nature of these mechanisms in relation to maturation remains unclear. Theoretically, symptom remission occurs via brain compensation-reorganization, and/or normalization-convergence, with a possible fixed anomaly 'scar' or enduring neurological trait-all of which may concurrently arise in different brain regions [4]. In a double dissociative neurodevelopmental model of ADHD, the underlying neural mechanisms that control onset are distinct from those that drive remission [5]. Thus, onset can be characterized by dysfunctional subcortical structures remaining static throughout life, while remission may be separately associated with brain (particularly prefrontal cortex) maturation and compensation $[4,6,7]$. The theory that maturing frontal cortical regions compensate for initial childhood ADHD emergence via top-down regulatory processes, leading to eventual symptom remission, has been supported by magnetic resonance imaging (MRI) studies: Reduced symptom severity throughout development appears to correlate with prefrontal cortex maturation. White matter (WM) development in frontal-temporal areas subserving emotional and cognitive processes indeed continues to mature into early adulthood, coinciding with the typical age range of ADHD symptom remission [5,8-12]. Based on this model, it is possible to methodologically differentiate remitted from unaffected brains with MRI. Yet, previous neuroimaging studies have reported inconsistent resultsperhaps because of study-specific differences (e.g. analysis methods, cross-sectional cohorts, sample characteristics). Considering this disorder's neurodevelopmental component, sample age is especially important, making systematic longitudinal studies essential in deconstructing the etiological timeline of brain mechanisms in reference to remission. 

and direction of water molecules diffusing through brain tissue, reflecting the underlying architecture of axons and their ensheathing myelin. Diffusion tensor imaging (DTI) has been the most commonly used DWI method in ADHD studies, which have usually reported tensor-derived, voxel-wise measures like fractional anisotropy (FA). One follow-up case-control DTI investigation in men suggested that $A D H D$ is a lasting neurobiological trait irrespective of remission or persistence: Compared to those who did not have childhood ADHD, probands with both remittent and persistent ADHD showed widespread reduced FA three decades post-diagnosis [6]. Others showed that children who exhibited symptom improvement had the most FA anomalies at follow-up [13]. However, given ADHD's neurodevelopmental aspect, studying symptoms and brain tissues in late adolescence and early adulthood (as myelination continues) can give more relevant information about how remission is intertwined with maturation. While valuable, the few follow-up DTI reports to date were limited by categorical participant groups, sample characteristics (populations that were either pre-pubertal or well into adulthood), and only a single follow-up MRI measure-underscoring the need for more studies beyond the cross-sectional perspective.

A longitudinal design reveals temporal dynamics of underlying neurobiological processes and increases statistical power by reducing inter-subject variability. The NeurolMAGE study and its latest follow-up, DELTA, is a longitudinal cohort of ADHD-affected probands, their siblings, and unaffected controls from childhood to adulthood [10,14-17]. We previously demonstrated that, at two different time-points and in two partly overlapping NeurolMAGE samples, more improvement in combined ADHD and hyperactivity-impulsivity symptom scores over time were associated with lower FA at follow-up in an area where the left corticospinal tract (ICST) crosses the left superior longitudinal fasciculus (ISLF) $[10,17]$. In that same report, we also systematically demonstrated that symptom change is associated with neither baseline FA nor change in FA from baseline to follow-up. Symptom remission was counterintuitively and repeatedly associated with decreased FA later in life, whether from childhood to adolescence, or to early adulthood. Our longitudinal findings indicated divergent 
WM microstructure trajectories between individuals with persistent and remittent symptoms at a follow-up age range of $12-29$ years. Now, we build on those previous results on the downstream effect of symptom progression on WM microstructure by asking whether the same relationship exists in the same brain areas at a later time window, when the cohort is aged $18-34$ years (Figure 1).

Although in vivo WM microstructure has been most commonly studied through tensorderived metrics (e.g. FA), results from voxel-wise DTI-based methods can be unreliable or misleading in areas with complex fiber architecture [18]. Given that tensor-based reconstructions are an average across an entire voxel and that approximately $90 \%$ of voxels contain multiple fiber populations, we applied a high angular diffusion model: constrained spherical deconvolution [19]. Voxel-based methods that model crossing fibers (e.g. BEDPOSTX) only represent a subset of the full range of possible fiber orientation distributions (FODs), whereas constrained spherical deconvolution represents FODs as spherical harmonics, free to distinguish more or less arbitrary shapes [20]. Fixelbased analysis (FBA) applies the constrained spherical deconvolution model and can more accurately reconstruct a continuous FOD in both single- and multiple-fiber voxels-characterizing properties of each "fixel," or specific fiber population in a voxel [21-25]. Fixels can be statistically analyzed for fiberspecific indices of underlying physiology: fiber density (FD), a microstructural measure of the withinvoxel intra-axonal restricted compartment of a fiber population; fiber cross-section (FC), a macrostructural measure of the area perpendicular to the fiber orientation; and fiber density and cross-section (FDC), a combination of FD and FC [25]. Less FD can indicate axonal loss, while less FC can indicate macroscopic fiber atrophy [26-28]. FBA resolves crossing fibers more accurately as well as characterizes the microstructural and morphological, macrostructural properties of specific fiber populations.

One cross-sectional FBA showed that ADHD-affected children who had reduced fine motor competence also had lower WM microstructure in all three fixel metrics in the CST. These results suggest that cases had fewer and/or thinner CST axons, which may lead to reduced fiber bundle information transmission speed [29]. Despite the consistent clamor to resolve crossing fiber regions 
and FBA's evident advantages, there have been no other published FBA applications in people with ADHD to our knowledge. Furthermore, besides our prior research, there have been no other longitudinal follow-up DWI studies of WM in the course of ADHD. over approximately 15 years. We used a more recent multi-shell DWI fiber model and a new followup measurement at an older age range. We aimed to assess the time-lag between the course of ADHD symptomatology and WM microstructure in a priori models and regions-of-interest. Because our smaller sample size at an older age range is not suitable for a data-driven search to discover any new relevant regions or tracts, the present analyses were intended to further understand the nature of our previous results. To compare FBA metrics to our previous FA findings, our first follow-up analysis used the same exact sample as our most recent longitudinal DTI study [17]. We hypothesized that, like in our earlier findings, ADHD symptom improvement would be associated with lower follow-up WM microstructure in the ISLF and ICST.

\section{Methods}

\subsection{Participants} first-degree relatives, and healthy families in one initial wave: NeurolMAGE1 (W1) [14]. After an average of 3.7 years (standard deviation $[S D]=0.5$ years), those participants were invited back for a second acquisition: NeurolMAGE2 (W2). After a mean of 5.1 years (SD = 1.4 years), some individuals returned for another wave, DELTA (W3), which included only people who fulfilled full ADHD diagnostic criteria in at least one previous wave (Table 1 ). For the analyses here, we only included participants who had clinical data from at least two of the three waves and DWI data from W2 and/or W3 (Figure 1). For each time-point, there were no differences between the participants included in the current analyses and the complete sample in symptom severity, age, and sex $(p>0.12)$. 
Through the years, symptom scores and diagnoses varied through time and participant characteristics changed from wave to wave (Figure 2). Some individuals originally recruited as controls or unaffected siblings developed ADHD at a later time point and others recruited as ADHD participants remitted, further highlighting the complex, variable course of ADHD. Alternative to a case-control categorization, ADHD can be operationalized as a continuous trait $[30,31]$. In a previous crosssectional study, we systematically showed that, compared to categorical diagnoses, continuous symptom measures are more sensitive to diffusion-weighted brain features in this sample [16]. Thus, all models here used ADHD symptom scores, optimally capturing the dynamic and continuous nature of the ADHD spectrum throughout development in this longitudinal cohort.

\subsection{Clinical symptom measures}

For continuous measures of symptom dimension severity and in accordance with our previous report, we used raw combined Conners' Parent Rating Scale (CPRS) scores from W1 and W2, and Conners' Adult ADHD Rating Scale (CAARS) scores from W3 for hyperactivity-impulsivity (HI) and inattention (IA) $[17,32,33]$. Here, we define symptom change $(\Delta)$ as the Conners' score difference:

Baseline versus follow-up scores were always positively correlated with each other (Figure S1). A more positive $\Delta$ value indicates the worsening of symptoms, while a more negative $\Delta$ value indicates the improvement of symptoms over time. In this report, we refer to "symptom remission" dimensionally and not diagnostically, i.e. a decrease or improvement in symptom severity over time. At W1 and W2, we assessed history of comorbid disorders with the Kiddie Schedule for Affective Disorder and Schizophrenia Present and Lifetime Version (K-SADS-PL) semi-structured interview [34,35]. For children aged $<12$ years, the child's parents or the researchers assisted in completing the self-report questionnaires. Participants with elevated scores on $\geq 1$ of the K-SADS-PL screening questions had to complete a full supplement for each disorder. At W3 (all participants were aged $\geq 18$ years), we recorded history of comorbidity using the Structured Clinical Interview for DSM- 5 
Disorders (SCID-V) [36]. IQ was estimated using the vocabulary and block design subtests of the

Wechsler Intelligence Scale for Children (WISC-III) or Wechsler Adult Intelligence Scale (WAIS-III). We excluded one whole dataset from a participant who had an estimated IQ $<70$. Our final sample's demographic characteristics are summarized in Table 1.

\subsection{Diffusion-weighted imaging acquisition, pre-processing, and quality control}

At W2, single-shell DWI data were acquired with a 1.5-Tesla AVANTO scanner (Siemens,

Erlangen, Germany) equipped with an 8-channel receive-only phased-array head coil using the following parameters: echo time/repetition time $(T E / T R)=97 / 8500 \mathrm{~ms}$; GRAPPA-acceleration factor 2 ; voxel size $=2 \times 2 \times 2.2 \mathrm{~mm} ; b$-values $=0$ ( 5 volumes, interleaved $)$ and 1000 (60 directions) $\mathrm{s} / \mathrm{mm}^{2}$; twice refocused pulsed-gradient spin-echo EPI; no partial Fourier. More details of this MRI data acquisition have been described previously $[16,17]$. Because our models only included follow-up neuroimaging data as a further investigation of the aforementioned analyses, we did not include W1 DWI.

At W3, multi-shell DWI data were acquired with a 3-Tesla Prisma scanner (Siemens, Erlangen, Germany) equipped with a 32-channel receive-only phased-array head coil using the following parameters: $\mathrm{TE} / \mathrm{TR}=75 / 2940 \mathrm{~ms} ;$ multi-band acceleration factor $=3$, voxel size $=1.8 \mathrm{~mm}^{3} ; b$-values $=$ 0 (11 volumes, interleaved), 1250 (86 directions), and 2500 ( 85 directions) s/mm². according to recommended FBA protocols for multi-shell data [24,25]. Pre-processing included denoising and unringing, motion and distortion correction, and bias field correction [37-43]. We visually inspected all corrected diffusion images and excluded whole datasets if any motion or 177 distortion artefacts remained after pre-processing. After excluding 22 datasets, our final sample consisted of 154 total diffusion scans collected from 139 participants at $\mathrm{W} 2(\mathrm{~N}=99)$ and $\mathrm{W} 3(\mathrm{~N}=55)$. 


\subsection{Fixel-based analysis}

Following pre-processing, we computed two unique group average tissue response functions for W2: WM and cerebrospinal fluid (CSF) [44]. B0 images can be utilized like a second shell to estimate a CSF-specific response function for each participant [44]. By modeling distinct response functions for WM and CSF, we were able to enhance the signal from WM relative to CSF and include our single shell data in the multi-shell FBA pipeline. For W3, we calculate three response functions: WM, gray matter, and CSF [44]. We upsampled to $1.25 \mathrm{~mm}^{3}$ and performed multi-shell multi-tissue constrained spherical deconvolution on all images, resulting in a WM fiber orientation distribution (FOD) within each voxel $[22,45]$. Afterwards, we performed joint bias field correction and global intensity normalization for each of the multi-tissue compartment parameters [41]. We then separately generated two studyspecific FOD population templates for W2 and W3 using 40 unrelated participants from each wave per template. Symptom scores did not differ between the individuals included in the population templates, versus those of the overall samples $(P>0.06)$.

For each population template, we calculated the $F D, \log (F C)$, and $F D C(F D C=F D \cdot F C)$ for each participant across all fixels. Instead of $\mathrm{FC}$, we chose to calculate $\log (\mathrm{FC})$ so data would be centered around zero and normally distributed. The derivation of these fixel metrics, which are based on FOD lobe segmentation and subject-to-template registration warps, are described in detail elsewhere [46]. For each FOD template, we performed whole-brain fiber tractography and generated a fixel-fixel connectivity matrix from the whole-brain streamline tractograms.

To obtain each region-of-interest (left corticospinal tract: ICST; left superior longitudinal fasciculus: ISLFI, ISLFII, ISLFIII; right cingulum: rCG), we extracted the spherical harmonic peaks from each voxel of both FOD population templates. We then applied TractSeg, which is an automated convolutional neural network-based approach that directly segments tracts in fields of FOD peaks, circumventing any biases that may result from user-defined or atlas-based delineation [47]. Finally, we converted the resultant tractograms to fixel maps used as masks to constrain our search space during connectivity-based fixel enhancement [46] (Figure 3 and Figure S2). 


\subsection{Statistical analyses}

To control for the lack of independence in our sample due to siblings, we designed multi-level exchangeability blocks per wave and used FSL PALM to generate a set of 5000 permutations per wave $[48,49]$. Our blocks did not allow permutation between all individuals; instead, we constrained permutations at both the whole-block level (i.e. permute between families of the same size) and within-block level (i.e. permute within families) (Figure S3). We used each set as an input for its respective wave to define permutations in data shuffling during nonparametric testing. (GLMs) to separately test whether combined ADHD or $\mathrm{HI}$ symptom score change ( $\triangle$ score as independent variables) are associated with fixel metrics at follow-up (as dependent variables) [17]. follow-up - age baseline), age at baseline, sex, and head motion (framewise displacement) at follow-up. For the W2 FBA, follow-up was W2 and baseline was W1, while for the W3 FBA, follow-up was W3 and baseline was W2: fixel metric follow-up $\sim \Delta$ score + score $_{\text {baseline }}+\Delta$ age + age baseline + sex + head motion

As a secondary cross-sectional analysis in W3 only, using the same aforementioned methods, 


\section{Results}

1.092, standardized effect $[\mathrm{SE}]=0.044, p_{\mathrm{FWE}}=0.016$; Figure 4$)$. There were no other significant

(all $p_{\mathrm{FWE}} \geq 0.051 ;$ Table S1).

W3 log of fiber cross-section (log[FC]) in the ICST was significantly negatively associated with

$\Delta$ combined symptom score $\left(t_{\max }=3.775, \mathrm{SE}=0.051, p_{\mathrm{FWE}}=0.019 ;\right.$ Figure 4$)$. There were no other

\subsection{Association between white matter and symptoms at Wave 3 only}

None of the global or rCG WM microstructure metrics were significantly associated with HI,

IA, or combined CAARS score cross-sectionally (all $p_{\mathrm{FWE}} \geq 0.053$; Table S3).

\section{Discussion}

We conducted a unique study of WM microstructure and longitudinal ADHD symptom development between ages 9 and 34 years. Using the FBA framework, we discovered two findings in the ICST: (1) HI symptom improvement was associated with axonal expansion at follow-up, and (2) combined ADHD symptom improvement was associated with a larger total cross-sectional area at follow-up at a slightly later age-range. Initially, a previous voxel-wise analysis in an overlapping sample found that improved HI symptoms were associated with lower follow-up FA (W1, aged 9 - 26 years) 
years), and systematically applied and excluded specific models-ultimately replicating the same effects on follow-up FA in the same WM region [17]. Given the counterintuitive nature of these previous highly consistent results, the present analysis aimed to further understand the physiological origins and its dynamic nature in relation to maturation. Thus here, in the exact same sample (W1W2) and including yet a third DWI acquisition (W3, aged 18-34 years), using the more advanced FBA method, and employing the same GLMs in which we previously found significant voxel-wise effects, we have found increased FD in relation to $\mathrm{HI}$ remission, and increased FC in relation to combined symptom remission, in only the ICST and not the ISLF. In contrast to our previous finding using DTIbased methods, our current finding using FBA is more intuitive in the direction of its effect: Indices that are generally indicative of "stronger" fibers were associated with clinical improvements over time.

The fixel metrics we used for quantifying WM microstructure contain complementary information. FD is thought to be related to the microstructural properties of WM, whereas FC pertains to the macrostructural properties (cross-sectional area). FD is an estimate of the intracellular volume of fibers oriented in a particular direction. Higher FD at follow-up could result from developmental processes like axon diameter growth, or more axons occupying a given space [50]. In our W1-to-W2 analysis, greater ICST axonal density in individuals who became less hyperactive-impulsive over time suggests plasticity, or a greater ability to relay information, after symptom improvement. Furthermore, higher myelin content could decrease the water exchange between intra- and extraaxonal compartments, resulting in an apparent increase in the intra-axonal compartment's volume and, hence, an increase in FD [42,51]. FC measures the morphological macroscopic change in the cross-sectional area perpendicular to a fiber bundle (calculated during registration to the template image). In W2-to-W3, higher follow-up ICST cross-sectional area in individuals whose combined symptom score improved, again, suggests plasticity, greater myelination, or fiber bundle organization after symptom remission [25]. 

aforementioned FA analyses, they are not incompatible. In some cases, crossing fiber complexity can have an inverse correlation with FA, wherein greater complexity occurs when more fixels in a voxel have the same fiber density [52]. An analogous inverse association exists in our previous W1-to-W2 voxel-wise analysis, wherein less follow-up FA was associated with improved HI symptom score. Notably, our results were in the approximate location of where the ISLF and ICST cross, while our present fixel-wise results in the ICST seem to be absent from where the ISLF crosses this tract. Therefore, as we previously suggested, our tract-based spatial statistics results may have been due to the neuroanatomical location of the effects, which, when labeled with an atlas, were in an area where these tracts cross. Compared to our voxel-wise study, we presently accounted for crossing fibers better through FBA, as well as the specific, FOD-based segmentation of these tracts as separate regions-of-interest. Accordingly, symptom improvement over time can conceivably be associated with increased CST fiber maturation, which by our previous DTI methods may have appeared as reduced FA in voxels where a more dominant SLF crosses those corticospinal fibers. previous longitudinal effects, which suggests a dissociation in the WM tracts associated with crosssectional differences versus those that are associated with symptom remission. This dissociation points to the neurodevelopmental models of remission, which all predict atypical neural features in adults with persistent ADHD, but have different predictions about those with remittent symptoms: If symptom remission occurred via WM normalization, convergence, or passive delayed maturation, then we would have observed neurological alterations at follow-up in participants with persistent symptoms, but no differences between those with remittent symptoms and healthy controls. If, regardless of symptom trajectory, this disorder imparted an indelible mark or scar on the brain, then 
remittent symptoms, and only healthy controls would have been differentiated by our follow-up analyses. If symptom remission occurred via compensation or reorganization, then remitted brains would have differed from both the never affected and the persistent ADHD brains, but in different ways. The dissociation we observed in the tract that is important for symptom remission versus the tract that is important for symptom severity implies the last model of remission. According to our current findings, we tentatively suggest an interpretation consistent with our previous report: In a top-down fashion, remitters may have learned compensatory strategies to overcome symptoms as they aged, while persisters may have either learned disadvantageous strategies, other beneficial (but insufficiently effective) compensatory strategies, or none at all-leading to diverging WM development trajectories in specific brain regions in individuals with persistent ADHD symptoms (Figure S4).

Based on our longitudinal design, we postulate that different WM alteration patterns are associated with symptom trajectory in a tract-specific manner: HI symptom remission preceded ICST plasticity at 20 years median age (range $12-29$ years), and combined symptom remission preceded ICST plasticity at 26 years median age (range $18-34$ years). Perhaps our sample at a slightly younger age, in response to HI symptom improvement or learning new skills, gained more ICST fibers over time. Tract expansion could have been a compensatory mechanism to improve motor control, followed by more myelination of those fibers. Then, as our participants became slightly older, improvement in both dimensions may have led to greater ICST WM microstructure and improved motor control (Figure S5). We can speculate that improved IA (and related executive control) could help suppress $\mathrm{HI}$, leading to greater motor control evinced as larger FC at a later age. In our remitters, higher measures of ICST WM might also result from reorganization in other brain areas outside of the tracts we studied. Along with our previous study, we have again found that alterations in WM microstructure appear to follow symptom improvement. Speculatively, this suggests that WM changes may be a downstream result of ADHD symptom remission. 

points. Our approach using two separate follow-up analyses lent further characterization to the temporal dynamics of ADHD-WM microstructure interplay. Of particular concern given this disorder, we mitigated potential confounding effects of head motion through careful data screening, correction during preprocessing, and inclusion as a covariate in our models. Using multi-shell FBA, we demonstrated that WM-associated differences are fiber-specific even within regions of crossing fibers, and we were able to further characterize WM micro- and macrostructural properties. Nonetheless, a limitation of this study is that the first follow-up analysis included DWI data acquired with only one relatively low b-value at $1.5 \mathrm{~T}$, which may have precluded us from discovering effects in other tracts and/or symptom dimensions since higher b-value shells improve correspondence between FD estimates and intra-axonal signal fraction simulations by increasing extra-axonal signal suppression [53]. Second, our inability to replicate our previous cross-sectional tractography findings may stem from the different DWI analysis methods used; also, W3 included a comparatively smaller number of affected-only individuals, which reduced our statistical power to detect smaller cross-sectional effects in the cingulum. Third, our follow-up samples were prone to selection bias from attrition and our explicit selection criteria in W3, where we also used different instruments (CRPS vs. CAARS) and raters. Returning participants were different from those who participated only once. Finally, even in a longitudinal study, we cannot prove causality. ADHD symptom persistence is likely associated with many other factors in daily life, or medication, or comorbid symptomatology-and any combination of these could also contribute to neurological differences at follow-up. Given our small sample size, we used a priori regions-of-interest and models, but a larger whole-brain analysis would be a more sufficient test of which mechanisms of remission are at play. symptoms in relation to WM development. Defining the correlates and predictors of remission may eventually lead to an improved allocation of treatment resources for persistent or complicated ADHD. 
354 A better understanding of the underlying neural mechanisms of these changes in time can contribute to the promotion of favorable future perspectives for children and adolescents with this disorder.

\section{Funding, acknowledgments, and financial disclosures} researchers who collected the data. This study sample is from the NeurolMAGE project. NeuroIMAGE

is the longitudinal follow-up study of the Dutch part of the International Multisite ADHD Genetics

(IMAGE) project, which was a multi-site, international effort. NeurolMAGE was supported by an NWO been in the past 3 years a consultant to / member of advisory board of / and/or speaker for companies, and not a stock shareholder of any of these companies. He has no other financial or material support, including expert testimony, patents, royalties. All other authors report no biomedical financial interests or potential conflicts of interest.

\section{References}

[1] Faraone, S. V., Biederman, J. and Mick, E. (2006) The age-dependent decline of attention deficit hyperactivity disorder: A meta-analysis of follow-up studies. Psychological Medicine, 36, 159- 

https://doi.org/10.1038/nrdp.2015.20

[3] Sibley, M.H., Mitchell, J.T. and Becker, S.P. (2016) Method of adult diagnosis influences estimated persistence of childhood ADHD: a systematic review of longitudinal studies. The Lancet Psychiatry, Elsevier Ltd. 3, 1157-65. https://doi.org/10.1016/S2215-0366(16)30190-0

[4] Sudre, G., Mangalmurti, A. and Shaw, P. (2018) Growing out of attention deficit hyperactivity disorder: Insights from the 'remitted' brain. Neuroscience and Biobehavioral Reviews, 94, 198209. https://doi.org/10.1016/j.neubiorev.2018.08.010

[5] Halperin, J.M. and Schulz, K.P. (2006) Revisiting the role of the prefrontal cortex in the pathophysiology of attention-deficit/hyperactivity disorder. Psychological Bulletin, Department of Psychology, Queens College of the City University of New York, Flushing, NY 11367, USA. jeffrey.halperin@qc.cuny.edu. 132, 560-81. https://doi.org/10.1037/00332909.132.4.560

[6] Cortese, S, Klein, R.G., Mannuzza, S., Ramos-olazagasti, Maria A, Castellanos, F., Cortese, S., Imperati, D., Zhou, J. et al. (2013) White Matter Alterations at 33-Year Follow-Up in Adults with Childhood Attention-Deficit/Hyperactivity Disorder. Biol Psychiatry, 74, 591-8. https://doi.org/10.1016/j.biopsych.2013.02.025.White

[7] Shaw, P., Sudre, G., Wharton, A., Weingart, D., Sharp, W. and Sarlls, J. (2015) White matter microstructure and the variable adult outcome of childhood attention deficit hyperactivity

[8] Clerkin, S.M., Schulz, K.P., Berwid, O.G., Fan, J., Newcorn, J.H., Tang, C.Y. et al. (2013) Thalamocortical activation and connectivity during response preparation in adults with persistent and disorder. Neuropsychopharmacology, Nature Publishing Group. 40, 746-54. https://doi.org/10.1038/npp.2014.241

remitted ADHD. American Journal of Psychiatry

170, 1011-9. https://doi.org/10.1176/appi.ajp.2013.12070880

[9] Shaw, P., Malek, M., Watson, B., Greenstein, D., De Rossi, P. and Sharp, W. (2013) Trajectories 
of cerebral cortical development in childhood and adolescence and adult attentiondeficit/hyperactivity disorder. Biological Psychiatry, Elsevier. 74, 599-606. https://doi.org/10.1016/j.biopsych.2013.04.007

[10] Francx, W., Zwiers, M.P., Mennes, M., Oosterlaan, J., Heslenfeld, D., Hoekstra, P.J. et al. (2015) White matter microstructure and developmental improvement of hyperactive/impulsive symptoms in attention-deficit/hyperactivity disorder. Journal of Child Psychology and Psychiatry and Allied Disciplines, Karakter Child and Adolescent Psychiatry University Centre, Nijmegen, The Netherlands. 56, 1289-97. https://doi.org/10.1111/jcpp.12379

[11] Cheung, C.H.M., Rijdijk, F., McLoughlin, G., Faraone, S. V, Asherson, P. and Kuntsi, J. (2015) Childhood predictors of adolescent and young adult outcome in ADHD. Journal of Psychiatric Research, 62, 92-100. https://doi.org/10.1016/j.jpsychires.2015.01.011

[12] Lebel, C. and Deoni, S. (2018) The development of brain white matter microstructure. Neurolmage, 182, 207-18. https://doi.org/10.1016/j.neuroimage.2017.12.097

[13] Sudre, G., Sharp, W., Kundzicz, P., Bouyssi-Kobar, M., Norman, L., Choudhury, S. et al. (2020) Predicting the course of ADHD symptoms through the integration of childhood genomic, neural, and cognitive features. Molecular Psychiatry, Springer US. https://doi.org/10.1038/s41380-020-00941-x

[14] von Rhein, D., Mennes, M., van Ewijk, H., Groenman, A.P., Zwiers, M.P., Oosterlaan, J. et al. (2015) The NeurolMAGE study: a prospective phenotypic, cognitive, genetic and MRI study in children with attention-deficit/hyperactivity disorder. Design and descriptives. European Child and Adolescent Psychiatry, Centre for Cognitive Neuroscience, Donders Institute for Brain, Cognition and Behaviour, Radboud University Medical Centre, Nijmegen, The Netherlands, Daniel.vonRhein@gmail.com. 24, 265-81. https://doi.org/10.1007/s00787-014-0573-4

[15] van Ewijk, H., Heslenfeld, D.J., Zwiers, M.P., Faraone, S. V., Luman, M., Hartman, C.A. et al. (2014) Different mechanisms of white matter abnormalities in attention-deficit/ hyperactivity disorder: A diffusion tensor imaging study. Journal of the American Academy of Child and 
Adolescent Psychiatry, Elsevier Inc. 53, 790-799.e3. https://doi.org/10.1016/j.jaac.2014.05.001

[16] Damatac, C.G., Chauvin, R.J.M., Zwiers, M.P., van Rooij, D., Akkermans, S.E.A., Naaijen, J. et al. (2020) White Matter Microstructure in Attention-Deficit/Hyperactivity Disorder: A Systematic Tractography Study in 654 Individuals. Biological Psychiatry: Cognitive Neuroscience and Neuroimaging, Elsevier Inc. 1-10. https://doi.org/10.1016/j.bpsc.2020.07.015

[17] Leenders, A. E. M. \& Damatac, C.G., Soheili-Nezhad, S., Chauvin, R.J.M., Mennes, M.J.J., Zwiers, M.P., Rooij, D. et al. (2021) Associations between attention-deficit hyperactivity disorder (ADHD) symptom remission and white matter microstructure: A longitudinal analysis. JCPP Advances, 1-11. https://doi.org/10.1002/jcv2.12040

[18] Seehaus, A., Roebroeck, A., Bastiani, M., Fonseca, L., Bratzke, H., Lori, N. et al. (2015) Histological validation of high-resolution DTI in human post mortem tissue. Frontiers in Neuroanatomy, 9. https://doi.org/10.3389/fnana.2015.00098

[19] Jeurissen, B., Leemans, A., Tournier, J.D., Jones, D.K. and Sijbers, J. (2013) Investigating the prevalence of complex fiber configurations in white matter tissue with diffusion magnetic resonance imaging. Human Brain Mapping, 34, 2747-66. https://doi.org/10.1002/hbm.22099

[20] Tournier, J.D., Calamante, F., Gadian, D.G. and Connelly, A. (2004) Direct estimation of the fiber orientation density function from diffusion-weighted MRI data using spherical deconvolution. Neurolmage, 23, 1176-85. https://doi.org/10.1016/j.neuroimage.2004.07.037

[21] Schilling, K.G., Janve, V., Gao, Y., Stepniewska, I., Landman, B.A. and Anderson, A.W. (2018) Histological validation of diffusion MRI fiber orientation distributions and dispersion. Neurolmage, 165, 200-21. https://doi.org/10.1016/j.neuroimage.2017.10.046

[22] Tournier, J.-D., Calamante, F. and Connelly, A. (2007) Robust determination of the fibre orientation distribution in diffusion MRI: Non-negativity constrained super-resolved spherical deconvolution. 
[23] Tournier, J.-D., Calamante, F. and Connelly, A. (2013) Determination of the appropriate b value and number of gradient directions for high-angular-resolution diffusion-weighted imaging. NMR in Biomedicine, England. 26, 1775-86. https://doi.org/10.1002/nbm.3017

[24] Tournier, J.D., Smith, R., Raffelt, D., Tabbara, R., Dhollander, T., Pietsch, M. et al. (2019) MRtrix3: A fast, flexible and open software framework for medical image processing and visualisation. Neurolmage, Elsevier Ltd. 202, 116137. https://doi.org/10.1016/j.neuroimage.2019.116137

[25] Raffelt, D.A., Tournier, J.-D.D., Smith, R.E., Vaughan, D.N., Jackson, G., Ridgway, G.R. et al. (2017) Investigating white matter fibre density and morphology using fixel-based analysis. Neurolmage, Elsevier. 144, 58-73. https://doi.org/10.1016/j.neuroimage.2016.09.029

[26] Gajamange, S., Raffelt, D., Dhollander, T., Lui, E., van der Walt, A., Kilpatrick, T. et al. (2018) Fibre-specific white matter changes in multiple sclerosis patients with optic neuritis. Neurolmage: Clinical, 17, 60-8. https://doi.org/10.1016/j.nicl.2017.09.027

[27] Mito, R., Raffelt, D., Dhollander, T., Vaughan, D.N., Tournier, J.D., Salvado, O. et al. (2018) Fibrespecific white matter reductions in Alzheimer's disease and mild cognitive impairment. Brain, 141, 888-902. https://doi.org/10.1093/brain/awx355

[28] Rojas-Vite, G., Coronado-Leija, R., Narvaez-Delgado, O., Ramírez-Manzanares, A., Marroquín, J.L., Noguez-Imm, R. et al. (2019) Histological validation of per-bundle water diffusion metrics within a region of fiber crossing following axonal degeneration. Neurolmage, 201, 116013. https://doi.org/https://doi.org/10.1016/j.neuroimage.2019.116013

[29] Hyde, C., Fuelscher, I., Sciberras, E., Efron, D., Anderson, V.A. and Silk, T. (2020) Understanding motor difficulties in children with ADHD: A fixel-based analysis of the corticospinal tract. Progress in Neuro-Psychopharmacology and Biological Psychiatry, Elsevier. 105, 110125. https://doi.org/10.1016/j.pnpbp.2020.110125

[30] Lahey, B.B. and Willcutt, E.G. (2010) Predictive Validity of a Continuous Alternative to Nominal Subtypes of Attention-Deficit/Hyperactivity Disorder for DSM - V. Journal of Clinical Child \& 
[31] Marcus, D.K. and Barry, T.D. (2011) Does attention-deficit/hyperactivity disorder have a dimensional latent structure? A taxometric analysis. Journal of Abnormal Psychology, 120, 427-42. https://doi.org/10.1037/a0021405

[32] Conners, C.K., Sitarenios, G., Parker, J.D.A. and Epstein, J.N. (1998) The revised Conners' Parent Rating Scale (CPRS-R): factor structure, reliability, and criterion validity. Journal of Abnormal

[33] Conners, C.K., Erhardt, D., Epstein, J.N., Parker, J.D.A., Sitarenios, G. and Sparrow, E. (1999)

[34] Kaufman, J., Birmaher, B., Brent, D., Rao, U., Flynn, C., Moreci, P. et al. (1997) Schedule for Affective Disorders and Schizophrenia for School-Age Children-Present and Lifetime Version (K-SADS-PL): initial reliability and validity data. Journal of the American Academy of Child and

Adolescent Psychiatry, United States. 36, 980-8. https://doi.org/10.1097/00004583$199707000-00021$ SCID-5-S: Gestructureerd Klinisch Interview Voor DSM-5 Syndroomstoornissen [Internet].

[35] Donker, T., Comijs, H., Cuijpers, P., Terluin, B., Nolen, W., Zitman, F. et al. (2010) The validity of the Dutch K10 and extended K10 screening scales for depressive and anxiety disorders. Psychiatry Research,. https://doi.org/10.1016/j.psychres.2009.01.012 Boom.

[37] Zwiers, M.P. (2010) Neurolmage Patching cardiac and head motion artefacts in diffusionweighted images. Neurolmage, Elsevier Inc. 53, 565-75. https://doi.org/10.1016/j.neuroimage.2010.06.014

[38] Veraart, J., Novikov, D.S., Christiaens, D., Ades-aron, B., Sijbers, J. and Fieremans, E. (2016) Denoising of diffusion MRI using random matrix theory. Neurolmage,. 
[39] Andersson, J.L.R. and Sotiropoulos, S.N. (2016) An integrated approach to correction for offresonance effects and subject movement in diffusion MR imaging. Neurolmage,. https://doi.org/10.1016/j.neuroimage.2015.10.019

[40] Tustison, N.J., Avants, B.B., Cook, P.A., Zheng, Y., Egan, A., Yushkevich, P.A. et al. (2010) N4ITK: Improved N3 bias correction. IEEE Transactions on Medical Imaging,. https://doi.org/10.1109/TMI.2010.2046908

[41] Raffelt, D., Dhollander, T., Tournier, J.D., Tabbara, R., Smith, R.E., Pierre, E. et al. (2017) Bias field correction and intensity normalisation for quantitative analysis of apparent fiber density. Proc Intl Soc Mag Reson Med,.

[42] Raffelt, D., Tournier, J.D., Rose, S., Ridgway, G.R., Henderson, R., Crozier, S. et al. (2012) Apparent Fibre Density: A novel measure for the analysis of diffusion-weighted magnetic resonance images. Neurolmage, Elsevier Inc.

59, 3976-94.

[43] Kellner, E., Dhital, B., Kiselev, V.G. and Reisert, M. (2016) Gibbs-ringing artifact removal based https://doi.org/10.1016/j.neuroimage.2011.10.045 on local subvoxel-shifts. Magnetic Resonance in Medicine, https://doi.org/10.1002/mrm.26054

[44] Dhollander, T., Raffelt, D. and Connelly, A. (2016) Unsupervised 3-tissue response function estimation from single-shell or multi-shell diffusion MR data without a co-registered T1 image.

[45] Jeurissen, B., Tournier, J.D., Dhollander, T., Connelly, A. and Sijbers, J. (2014) Multi-tissue constrained spherical deconvolution for improved analysis of multi-shell diffusion MRI data. Neurolmage, Elsevier Inc. 103, 411-26. https://doi.org/10.1016/j.neuroimage.2014.07.061 Connectivity-based fixel enhancement: Whole-brain statistical analysis of diffusion MRI measures in the presence of crossing fibres. Neurolmage, Elsevier B.V. 117, 40-55. https://doi.org/10.1016/j.neuroimage.2015.05.039 
[47] Wasserthal, J., Neher, P. and Maier-Hein, K.H. (2018) TractSeg - Fast and accurate white matter tract segmentation. Neurolmage, Elsevier Ltd. 183, 239-53. https://doi.org/10.1016/j.neuroimage.2018.07.070

[48] Winkler, A.M., Webster, M.A., Vidaurre, D., Nichols, T.E. and Smith, S.M. (2015) Multi-level block permutation. Neurolmage, The Authors. 123, 253-68. https://doi.org/10.1016/j.neuroimage.2015.05.092

[49] Winkler, A.M., Ridgway, G.R., Webster, M.A., Smith, S.M. and Nichols, T.E. (2014) Permutation inference for the general linear model. Neurolmage, The Authors. 92, 381-97. https://doi.org/10.1016/j.neuroimage.2014.01.060

[50] Genc, S., Smith, R.E., Malpas, C.B., Anderson, V., Nicholson, J.M., Efron, D. et al. (2018) Development of white matter fibre density and morphology over childhood: A longitudinal fixel-based analysis. Neurolmage, Elsevier Ltd. 183, 666-76. https://doi.org/10.1016/j.neuroimage.2018.08.043

[51] Pannek, K., Fripp, J., George, J.M., Fiori, S., Colditz, P.B., Boyd, R.N. et al. (2018) Fixel-based analysis reveals alterations is brain microstructure and macrostructure of preterm-born infants at term equivalent age. Neurolmage: Clinical, Elsevier. 18, 51-9. https://doi.org/10.1016/j.nicl.2018.01.003

[52] Grazioplene, R.G., Bearden, C.E., Subotnik, K.L., Ventura, J., Haut, K., Nuechterlein, K.H. et al. (2018) Connectivity-enhanced diffusion analysis reveals white matter density disruptions in first episode and chronic schizophrenia. Neurolmage: Clinical, Elsevier. 18, 608-16. https://doi.org/10.1016/j.nicl.2018.02.015

[53] Genc, S., Tax, C.M.W., Raven, E.P., Chamberland, M., Parker, G.D. and Jones, D.K. (2020) Impact of b-value on estimates of apparent fibre density. Human Brain Mapping, 41, 2583-95. https://doi.org/10.1002/hbm.24964 


\begin{tabular}{|c|c|c|c|c|c|c|c|c|}
\hline & \multirow{2}{*}{\multicolumn{2}{|c|}{$\begin{array}{l}\text { Wave } 1 \\
N=119\end{array}$}} & \multirow{2}{*}{\multicolumn{2}{|c|}{$\begin{array}{l}\text { Wave } 2 \\
N=135\end{array}$}} & \multirow{2}{*}{\multicolumn{2}{|c|}{$\begin{array}{l}\text { Wave } 3 \\
N=55\end{array}$}} & \multirow[b]{3}{*}{$\begin{array}{c}\text { Test } \\
\text { Statistic }\end{array}$} & \multirow[b]{3}{*}{ (p) } \\
\hline & & & & & & & & \\
\hline & Mean & (SD) & Mean & (SD) & Mean & (SD) & & \\
\hline Age, years & 16.98 & $(3.47)$ & 20.22 & $(3.48)$ & 24.82 & $(4.07)$ & & \\
\hline Sex, female & $N=48$ & $40 \%$ & $N=53$ & $39 \%$ & $N=19$ & $35 \%$ & $\chi^{2}{ }_{3}=1.51$ & $(0.68)$ \\
\hline Estimated $\mathrm{IQ}^{a}$ & 98.41 & $(14.81)$ & 105 & $(16.82)$ & 102.35 & (14.17) & $F_{2,192}=2.23$ & $(0.11)$ \\
\hline $\begin{array}{l}\text { Head motion, } \\
\text { framewise } \\
\text { displacement }\end{array}$ & 0.47 & $(0.17)$ & 0.53 & $(0.42)$ & 0.82 & $(0.17)$ & $F_{2,298}=24.71$ & $\left(<10^{-10}\right)$ \\
\hline Handedness, right & $N=96$ & $81 \%$ & $N=111$ & $82 \%$ & $N=45$ & $82 \%$ & $\chi^{2}{ }_{3}=4.61$ & $(0.20)$ \\
\hline $\begin{array}{l}\text { Medication ever } \\
\text { used, yes }{ }^{b, c}\end{array}$ & $N=66$ & $56 \%$ & $N=45$ & $33 \%$ & $N=42$ & $76 \%$ & $\chi^{2}{ }_{3}=32.03$ & $\left(<10^{-6}\right)$ \\
\hline \multicolumn{9}{|l|}{$\begin{array}{l}\text { Symptom raw } \\
\text { score by diagnostic } \\
\text { group }^{d}\end{array}$} \\
\hline Combined score ${ }^{e}$ & 13.03 & $(12.33)$ & 12.96 & $(13.05)$ & 20.53 & $(9.34)$ & & \\
\hline $\begin{array}{l}\text { Hyperactivity- } \\
\text { impulsivity score }\end{array}$ & 4.94 & (5.73) & 4.71 & $(5.68)$ & 10.85 & (5.38) & & \\
\hline Inattention score & 8.05 & (7.51) & 7.55 & (7.45) & 9.67 & $(4.85)$ & & \\
\hline \multicolumn{9}{|l|}{$\begin{array}{l}\text { Comorbidity } \\
\text { diagnosis }\end{array}$} \\
\hline Anxiety disorder & $N=2$ & $2 \%$ & $N=2$ & $1 \%$ & $N=1$ & $2 \%$ & $\chi^{2}{ }_{3}=57.344$ & $\left(<10^{-11}\right)$ \\
\hline $\begin{array}{l}\text { Avoidant } \\
\text { personality disorder }\end{array}$ & $N=1$ & $1 \%$ & $N=2$ & $1 \%$ & $N=0$ & $0 \%$ & $\chi^{2}{ }_{3}=81.886$ & $\left(<10^{-15}\right)$ \\
\hline Conduct disorder & $N=2$ & $2 \%$ & $N=2$ & $1 \%$ & $N A$ & $N A$ & $\chi^{2}{ }_{2}=55.107$ & $\left(<10^{-11}\right)$ \\
\hline Major depression & $N=1$ & $1 \%$ & $N=1$ & $1 \%$ & $N=4$ & $7 \%$ & $\chi^{2}{ }_{3}=57.435$ & $\left(<10^{-11}\right)$ \\
\hline $\begin{array}{l}\text { Oppositional } \\
\text { defiant disorder }\end{array}$ & $N=15$ & $13 \%$ & $N=16$ & $12 \%$ & $N A$ & $N A$ & $\chi_{2}^{2}=7.8901$ & (7.89) \\
\hline Panic disorder & $N=0$ & $0 \%$ & $N=1$ & $1 \%$ & $N=0$ & $0 \%$ & $\chi^{2}{ }_{3}=137.76$ & $\left(<10^{-15}\right)$ \\
\hline \multicolumn{9}{|l|}{ Substance use } \\
\hline Alcohol & $N=19$ & $16 \%$ & $N=21$ & $16 \%$ & $N=7$ & $13 \%$ & $\chi^{2}{ }_{3}=6.2621$ & $(0.10)$ \\
\hline Tobacco & $N=43$ & $36 \%$ & $N=45$ & $33 \%$ & $N=12$ & $22 \%$ & $\chi^{2}{ }_{3}=5.1952$ & $(0.16)$ \\
\hline Cannabis or hash & $N=22$ & $18 \%$ & $N=23$ & $17 \%$ & $N=6$ & $11 \%$ & $\chi^{2}{ }_{3}=6.8817$ & $(0.08)$ \\
\hline Other drugs & $N=6$ & $5 \%$ & $N=6$ & $4 \%$ & $N=2$ & $4 \%$ & $\chi^{2}{ }_{3}=23.734$ & $\left(<10^{-4}\right)$ \\
\hline
\end{tabular}


565 Table 1. Demographic and clinical characteristics of participants at Wave 1 (W1), Wave 2 (W2), and Wave 3 (W3) with mean and standard deviation (or numerical count and percentage). W3 included only those who fulfilled full ADHD diagnostic criteria in at least one previous wave. Values reported here are for all participants in the final sample after all quality control $(N=139)$.

${ }^{a}$ IQ was estimated using the vocabulary and block design subtests of the Wechsler Intelligence Scale for Children or Wechsler Adult Intelligence Scale.

${ }^{b}$ Medication ever used: Whether or not participants had ever taken ADHD medication.

c Medications: Ritalin (methylphenidate), Concerta (methylphenidate), Strattera (atomoxetine), and any other ADHD medication. The majority of patients were taking prescription medication for ADHD, mostly methylphenidate or atomoxetine.

${ }^{d}$ Symptom scores in W1 and W2 were collected via Conners' Parent Rating Scale, and W3 scores were collected via the Conners' Adult ADHD Rating Scale.

${ }^{e}$ Combined symptom score: Sum of hyperactivity-impulsivity and inattention scores. 


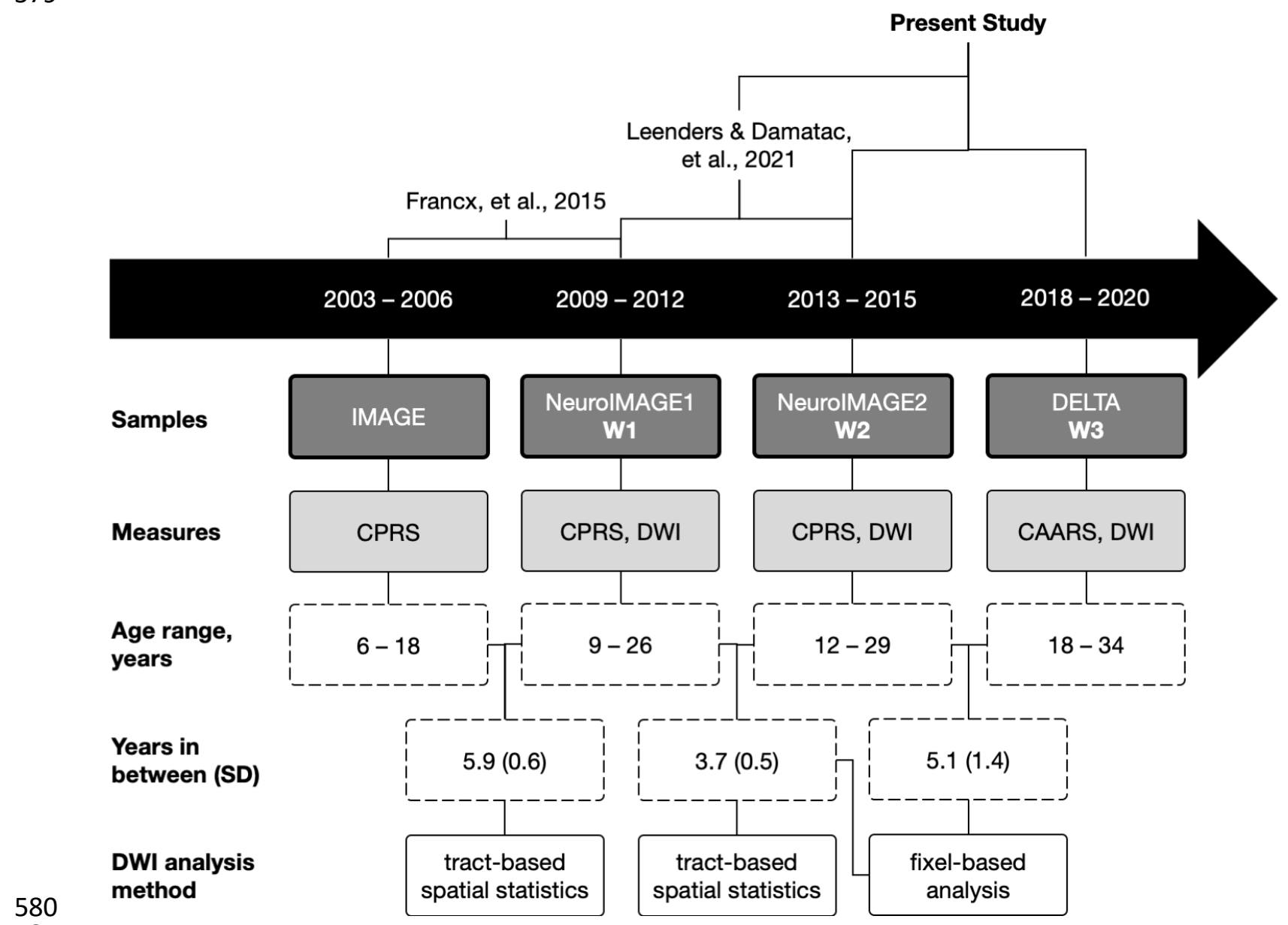

Figure 1. Schematic of how this study chronologically relates to previous studies, the samples included in each, relevant clinical and neuroimaging measurements, study sample age ranges, mean years (standard deviation) in between each acquisition wave, and the analysis methods used. The present study is a fixel-based analysis of W1 to W2 and W2 to W3, using only the models in which we found significant effects in a previous voxel-wise tract-based spatial statistical analysis of W1 to W2. 

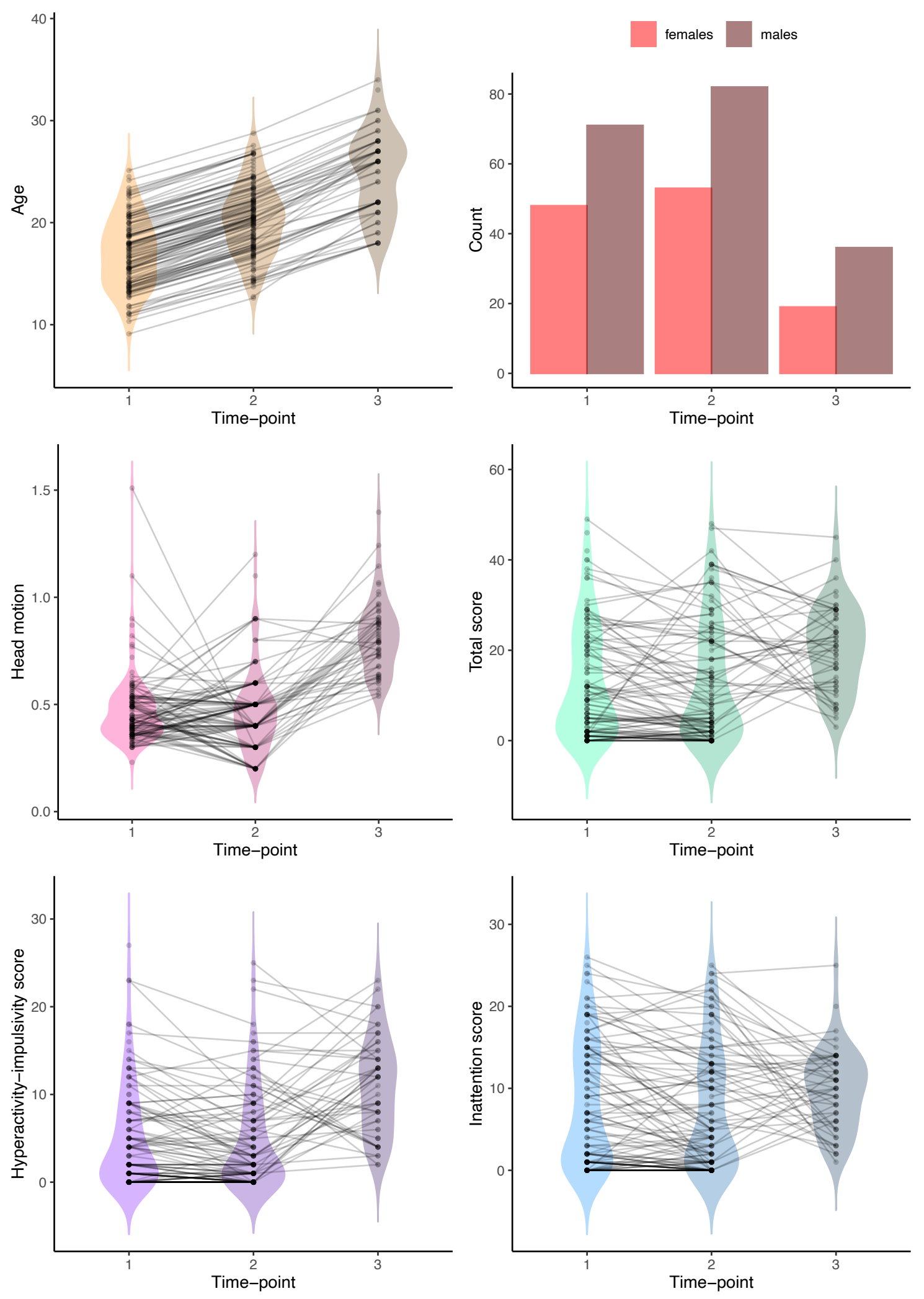

Figure 2. Change in participant characteristics from Wave 1, to Wave 2, to Wave 3. Longitudinal data points are connected by a line. Note that participants at W3 were selected on the basis of their history of ADHD diagnosis, so W3 tends to differ quite markedly from the other two waves, which also include never-affected controls. This conceals the typical pattern of average symptom remission that would be expected in a follow-up study without this selection criterion. 


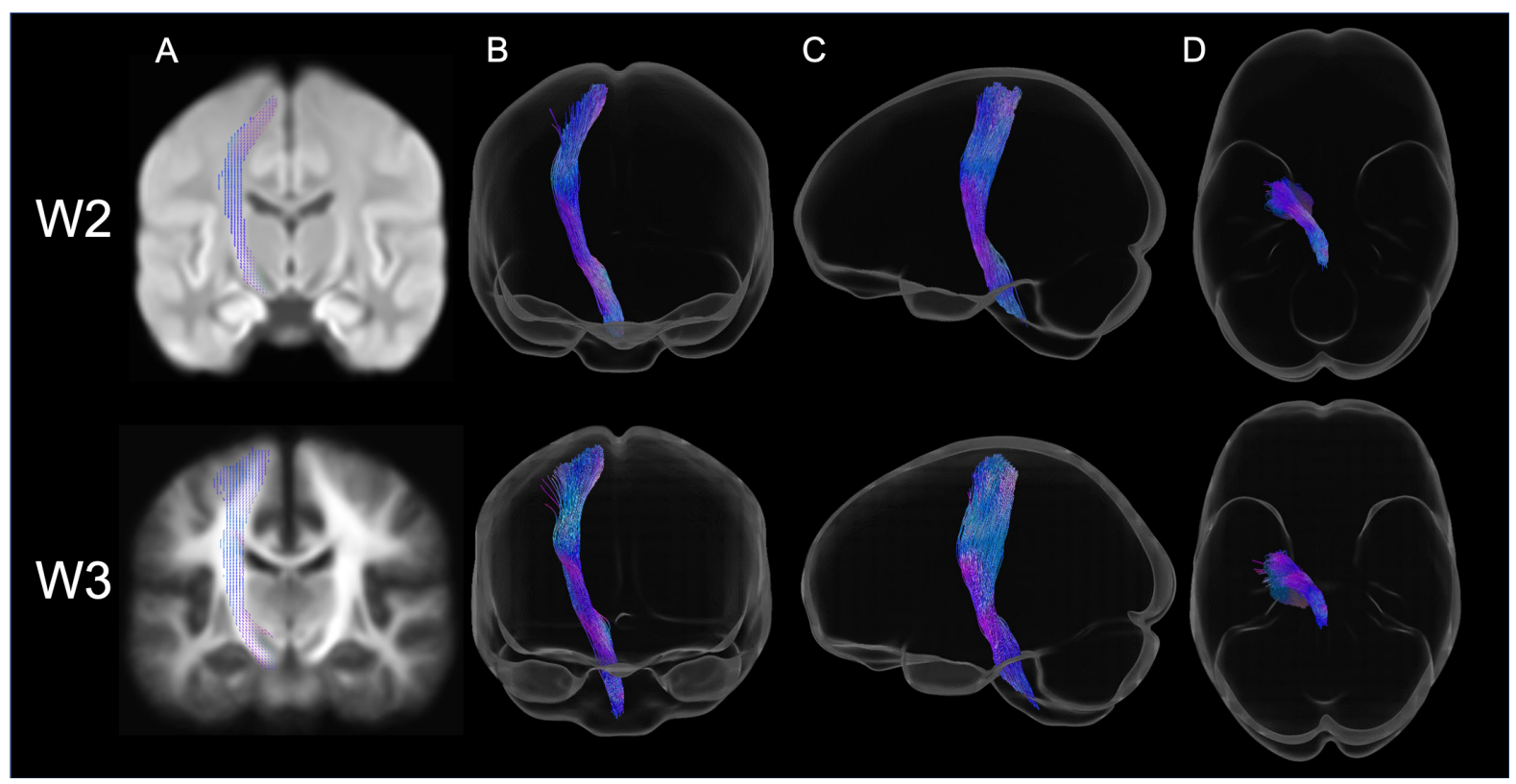

Figure 3. Tract-specific region-of-interest masks of the left corticospinal tract for Wave 2 (top) and Wave 3 (bottom) colored by direction (red: left-right, green: anterior-posterior, blue: inferiorsuperior). (A) Fixel mask overlaid on a single representative coronal slice of the study-specific white matter fiber orientation distribution template. Template contrast was adjusted and fixels have been thickened for visualization. (B) Coronal, (C) sagittal, and (D) axial views of the tract reconstruction from TractSeg (applied to each fiber orientation distribution template) and displayed in glass brains for visualization. 


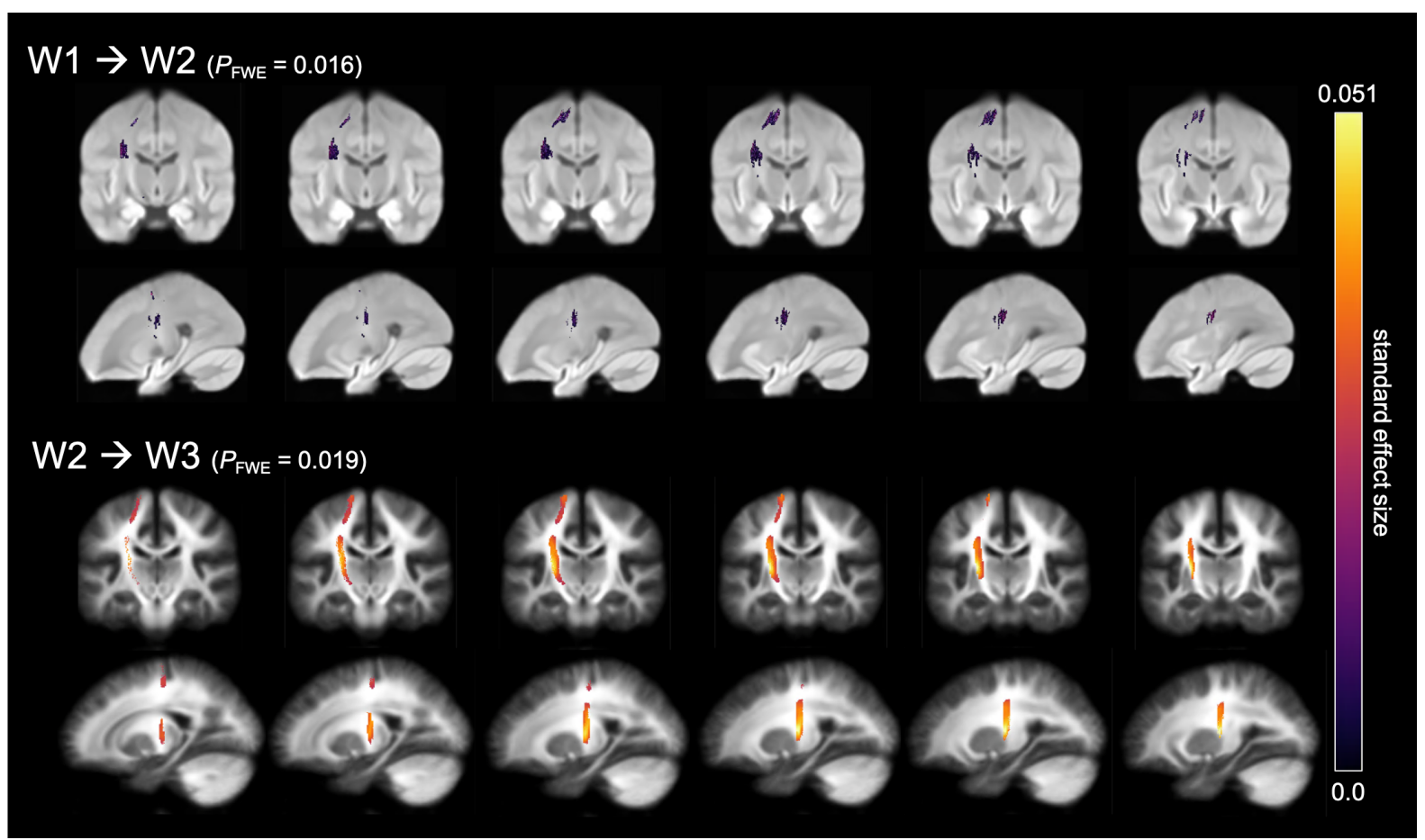

Figure 4. Symptom change may precede ICST WM microstructure plasticity. Top: Improvement of HI score is associated with more follow-up fiber density (FD). Bottom: Improvement of combined score is associated with more follow-up fiber cross-section (FC). Streamline segments have been cropped from the template tractogram to include only streamline points that correspond to significant fixels for this tract (FWE-corrected $p$-value $<0.05)$. Significant streamlines are colored by the standard effect size of ' $\triangle$ score' on 'FD at $W 2$ ' and ' $\log (\mathrm{FC})$ at $\mathrm{W}^{\prime}$ ' and displayed across coronal and sagittal slices of the study-specific white matter fiber orientation distribution templates. 


\begin{tabular}{|c|c|c|c|c|c|c|}
\hline Search space & $\begin{array}{l}\text { Dependent variable } \\
\text { Fixel-based metric }\end{array}$ & Contrast & $\begin{array}{c}\text { Independent variable } \\
\text { Symptom score change } \\
(\Delta=\text { W2 - W1) }\end{array}$ & $t_{\max }$ & Std. effect & $P_{\text {fWe }}$ \\
\hline \multirow{12}{*}{$\begin{array}{l}\text { Left } \\
\text { corticospinal } \\
\text { tract }\end{array}$} & \multirow{4}{*}{ Fiber density } & $(+)$ & combined & 0.858 & 0.011 & 0.165 \\
\hline & & $(-)$ & combined & 0.917 & 0.012 & 0.104 \\
\hline & & $(+)$ & $\mathrm{HI}$ & 0.949 & 0.038 & 0.225 \\
\hline & & $(-)$ & HI & 1.092 & 0.044 & $0.016 *$ \\
\hline & \multirow{4}{*}{ Fiber cross-section } & $(+)$ & combined & 1.846 & 0.023 & 0.591 \\
\hline & & $(-)$ & combined & 2.264 & 0.029 & 0.343 \\
\hline & & $(+)$ & $\mathrm{HI}$ & 1.909 & 0.076 & 0.627 \\
\hline & & $(-)$ & $\mathrm{HI}$ & 2.189 & 0.087 & 0.536 \\
\hline & \multirow{4}{*}{$\begin{array}{l}\text { Fiber density and cross- } \\
\text { section }\end{array}$} & $(+)$ & combined & 0.969 & 0.012 & 0.280 \\
\hline & & $(-)$ & combined & 0.828 & 0.011 & 0.439 \\
\hline & & $(+)$ & $\mathrm{HI}$ & 1.051 & 0.042 & 0.294 \\
\hline & & $(-)$ & $\mathrm{HI}$ & 0.948 & 0.038 & 0.083 \\
\hline \multirow{6}{*}{$\begin{array}{l}\text { Left superior } \\
\text { longitudinal } \\
\text { fasciculus I }\end{array}$} & \multirow{2}{*}{ Fiber density } & $(+)$ & combined & 2.238 & 0.028 & 0.496 \\
\hline & & $(-)$ & combined & 1.300 & 0.017 & 0.285 \\
\hline & \multirow{2}{*}{ Fiber cross-section } & $(+)$ & combined & 2.627 & 0.033 & 0.252 \\
\hline & & $(-)$ & combined & 1.759 & 0.023 & 0.474 \\
\hline & \multirow{2}{*}{$\begin{array}{l}\text { Fiber density and cross- } \\
\text { section }\end{array}$} & $(+)$ & combined & 2.483 & 0.032 & 0.112 \\
\hline & & $(-)$ & combined & 1.351 & 0.017 & 0.493 \\
\hline \multirow{6}{*}{$\begin{array}{l}\text { Left superior } \\
\text { longitudinal } \\
\text { fasciculus II }\end{array}$} & \multirow{2}{*}{ Fiber density } & $(+)$ & combined & 1.547 & 0.020 & 0.462 \\
\hline & & $(-)$ & combined & 1.462 & 0.019 & 0.682 \\
\hline & \multirow{2}{*}{ Fiber cross-section } & $(+)$ & combined & 1.987 & 0.025 & 0.319 \\
\hline & & $(-)$ & combined & 1.372 & 0.017 & 0.625 \\
\hline & \multirow{2}{*}{$\begin{array}{l}\text { Fiber density and cross- } \\
\text { section }\end{array}$} & $(+)$ & combined & 1.666 & 0.021 & 0.245 \\
\hline & & $(-)$ & combined & 1.304 & 0.017 & 0.777 \\
\hline \multirow{6}{*}{$\begin{array}{l}\text { Left superior } \\
\text { longitudinal } \\
\text { fasciculus III }\end{array}$} & \multirow{2}{*}{ Fiber density } & $(+)$ & combined & 1.464 & 0.019 & 0.762 \\
\hline & & $(-)$ & combined & 1.290 & 0.016 & 0.225 \\
\hline & \multirow{2}{*}{ Fiber cross-section } & $(+)$ & combined & 1.331 & 0.017 & 0.709 \\
\hline & & $(-)$ & combined & 3.121 & 0.040 & 0.051 \\
\hline & \multirow{2}{*}{$\begin{array}{l}\text { Fiber density and cross- } \\
\text { section }\end{array}$} & $(+)$ & combined & 1.766 & 0.022 & 0.863 \\
\hline & & $(-)$ & combined & 1.239 & 0.016 & 0.407 \\
\hline
\end{tabular}


bioRxiv preprint doi: https://doi.org/10.1101/2021.11.19.469248; this version posted November 22, 2021. The copyright holder for this

preprint (which was not certified by peer review) is the author/funder. All rights reserved. No reuse allowed without permission.

623

\begin{tabular}{|c|c|c|c|c|c|c|}
\hline Search space & $\begin{array}{l}\text { Dependent variable } \\
\text { Fixel-based metric }\end{array}$ & Contrast & $\begin{array}{c}\text { Independent variable } \\
\text { Symptom score change } \\
(\Delta=\text { W3 }- \text { W2) }\end{array}$ & $t_{\max }$ & Std. effect & $P_{\text {FWE }}$ \\
\hline \multirow{12}{*}{$\begin{array}{l}\text { Left } \\
\text { corticospinal } \\
\text { tract }\end{array}$} & \multirow{4}{*}{ Fiber density } & $(+)$ & combined & 1.288 & 0.017 & 0.612 \\
\hline & & $(-)$ & combined & 1.687 & 0.023 & 0.156 \\
\hline & & $(+)$ & $\mathrm{HI}$ & 1.344 & 0.036 & 0.636 \\
\hline & & $(-)$ & $\mathrm{HI}$ & 1.761 & 0.048 & 0.200 \\
\hline & \multirow{4}{*}{ Fiber cross-section } & $(+)$ & combined & 0.916 & 0.012 & 0.896 \\
\hline & & $(-)$ & combined & 3.775 & 0.051 & $0.019 *$ \\
\hline & & $(+)$ & $\mathrm{HI}$ & 0.911 & 0.025 & 0.960 \\
\hline & & $(-)$ & $\mathrm{HI}$ & 3.575 & 0.097 & 0.058 \\
\hline & \multirow{4}{*}{ Fiber density and cross-section } & $(+)$ & combined & 1.122 & 0.015 & 0.821 \\
\hline & & $(-)$ & combined & 1.776 & 0.024 & 0.093 \\
\hline & & $(+)$ & $\mathrm{HI}$ & 1.190 & 0.032 & 0.908 \\
\hline & & $(-)$ & $\mathrm{HI}$ & 1.800 & 0.049 & 0.141 \\
\hline \multirow{6}{*}{$\begin{array}{l}\text { Left superior } \\
\text { longitudinal } \\
\text { fasciculus I }\end{array}$} & \multirow{2}{*}{ Fiber density } & $(+)$ & combined & 1.632 & 0.022 & 0.428 \\
\hline & & $(-)$ & combined & 1.519 & 0.020 & 0.956 \\
\hline & \multirow{2}{*}{ Fiber cross-section } & $(+)$ & combined & 0.740 & 0.010 & 0.963 \\
\hline & & $(-)$ & combined & 2.863 & 0.038 & 0.169 \\
\hline & \multirow{2}{*}{ Fiber density and cross-section } & $(+)$ & combined & 1.727 & 0.023 & 0.413 \\
\hline & & $(-)$ & combined & 1.823 & 0.024 & 0.715 \\
\hline \multirow{6}{*}{$\begin{array}{l}\text { Left superior } \\
\text { longitudinal } \\
\text { fasciculus II }\end{array}$} & \multirow{2}{*}{ Fiber density } & $(+)$ & combined & 1.264 & 0.017 & 0.837 \\
\hline & & $(-)$ & combined & 1.247 & 0.017 & 0.882 \\
\hline & \multirow{2}{*}{ Fiber cross-section } & $(+)$ & combined & 0.882 & 0.012 & 0.978 \\
\hline & & $(-)$ & combined & 2.863 & 0.038 & 0.259 \\
\hline & \multirow{2}{*}{ Fiber density and cross-section } & $(+)$ & combined & 1.340 & 0.018 & 0.587 \\
\hline & & $(-)$ & combined & 1.305 & 0.017 & 0.730 \\
\hline \multirow{6}{*}{$\begin{array}{l}\text { Left superior } \\
\text { longitudinal } \\
\text { fasciculus III }\end{array}$} & \multirow[t]{2}{*}{ Fiber density } & $(+)$ & combined & 2.062 & 0.028 & 0.067 \\
\hline & & $(-)$ & combined & 1.572 & 0.021 & 0.340 \\
\hline & \multirow{2}{*}{ Fiber cross-section } & $(+)$ & combined & 1.450 & 0.019 & 0.778 \\
\hline & & $(-)$ & combined & 2.210 & 0.030 & 0.437 \\
\hline & \multirow{2}{*}{ Fiber density and cross-section } & $(+)$ & combined & 1.928 & 0.026 & 0.191 \\
\hline & & $(-)$ & combined & 1.734 & 0.023 & 0.516 \\
\hline
\end{tabular}


627

\begin{tabular}{|c|c|c|c|c|c|c|}
\hline Search space & $\begin{array}{l}\text { Dependent variable } \\
\text { Fixel-based metric }\end{array}$ & Contrast & $\begin{array}{l}\text { Independent variable } \\
\text { CAARS score } \\
\text { (W3) }\end{array}$ & $t_{\max }$ & Std. effect & $P_{\mathrm{FWE}}$ \\
\hline \multirow{18}{*}{ Whole brain } & \multirow{6}{*}{ Fiber density } & $(+)$ & combined & 3.011 & 0.045 & 0.562 \\
\hline & & $(-)$ & combined & 2.739 & 0.041 & 0.858 \\
\hline & & $(+)$ & $\mathrm{HI}$ & 3.195 & 0.082 & 0.501 \\
\hline & & $(-)$ & $\mathrm{HI}$ & 2.640 & 0.068 & 0.835 \\
\hline & & $(+)$ & IA & 3.120 & 0.095 & 0.053 \\
\hline & & $(-)$ & IA & 2.862 & 0.087 & 0.757 \\
\hline & \multirow{6}{*}{ Fiber cross-section } & $(+)$ & combined & 2.775 & 0.042 & 0.835 \\
\hline & & $(-)$ & combined & 4.446 & 0.067 & 0.261 \\
\hline & & $(+)$ & $\mathrm{HI}$ & 2.172 & 0.056 & 0.892 \\
\hline & & $(-)$ & $\mathrm{HI}$ & 4.218 & 0.109 & 0.173 \\
\hline & & $(+)$ & IA & 3.721 & 0.113 & 0.700 \\
\hline & & $(-)$ & IA & 3.749 & 0.114 & 0.362 \\
\hline & \multirow{6}{*}{$\begin{array}{l}\text { Fiber density and cross- } \\
\text { section }\end{array}$} & $(+)$ & combined & 3.027 & 0.046 & 0.678 \\
\hline & & $(-)$ & combined & 2.847 & 0.043 & 0.778 \\
\hline & & $(+)$ & $\mathrm{HI}$ & 3.111 & 0.080 & 0.864 \\
\hline & & $(-)$ & $\mathrm{HI}$ & 2.774 & 0.071 & 0.827 \\
\hline & & $(+)$ & IA & 3.166 & 0.096 & 0.119 \\
\hline & & $(-)$ & IA & 2.840 & 0.086 & 0.716 \\
\hline \multirow{6}{*}{$\begin{array}{l}\text { Right } \\
\text { cingulum } \\
\text { bundle }\end{array}$} & \multirow{2}{*}{ Fiber density } & $(+)$ & $\mathrm{HI}$ & 0.047 & 1.841 & 0.410 \\
\hline & & $(-)$ & $\mathrm{HI}$ & 0.043 & 1.678 & 0.315 \\
\hline & \multirow{2}{*}{ Fiber cross-section } & $(+)$ & $\mathrm{HI}$ & 0.763 & 0.020 & 0.980 \\
\hline & & $(-)$ & $\mathrm{HI}$ & 3.806 & 0.098 & 0.063 \\
\hline & \multirow{2}{*}{$\begin{array}{l}\text { Fiber density and cross- } \\
\text { section }\end{array}$} & $(+)$ & $\mathrm{HI}$ & 1.653 & 0.043 & 0.688 \\
\hline & & $(-)$ & $\mathrm{HI}$ & 1.764 & 0.045 & 0.172 \\
\hline
\end{tabular}



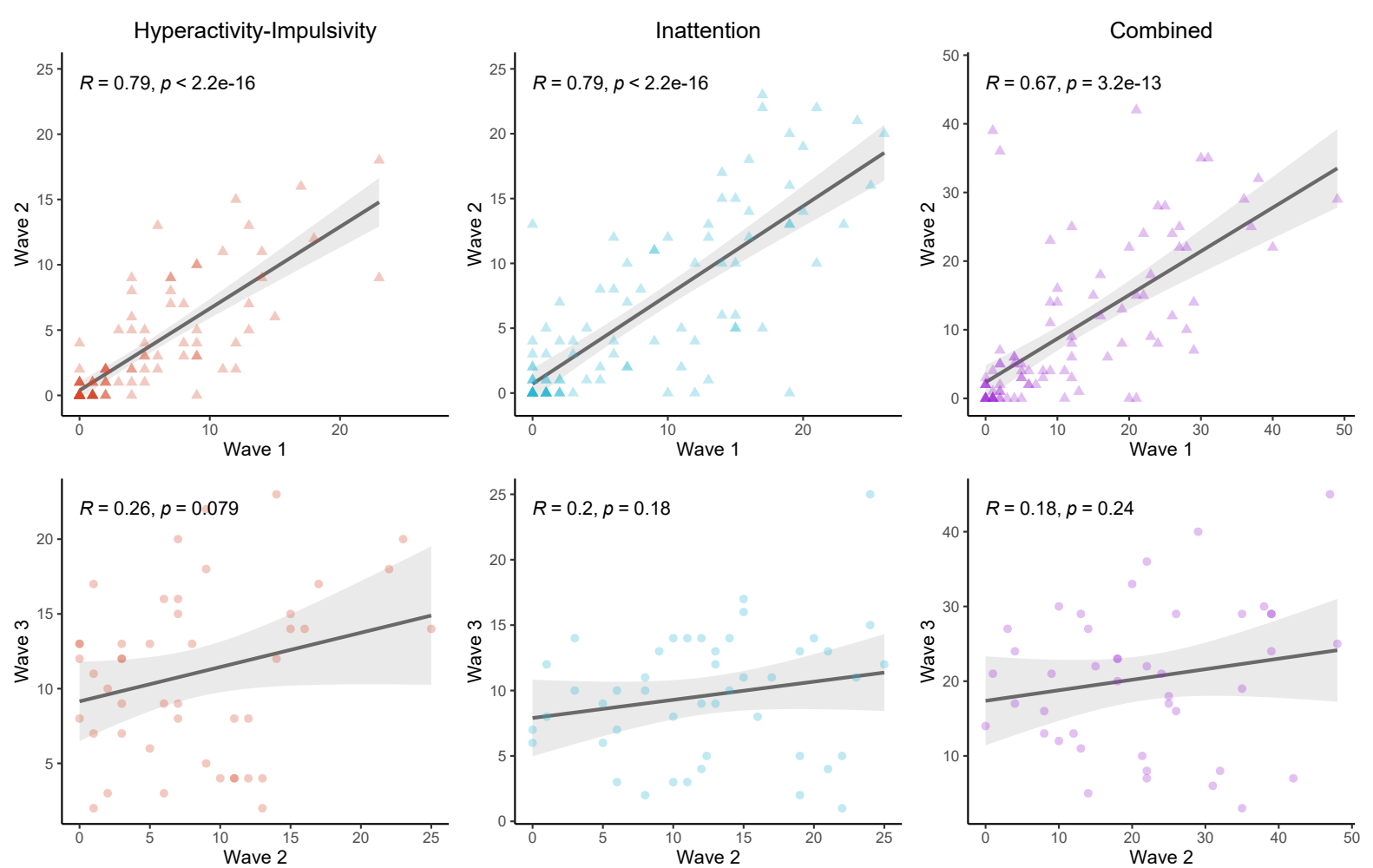

Figure S1. Correlation scatterplots of baseline against follow-up scores colored by dimension with $95 \%$ confidence intervals and Pearson correlation coefficients reported for each. Top row: Wave 1 associations with Wave 2 scores. Bottom row: Wave 2 associations with Wave 3 scores. Left column: Hyperactivity-impulsivity dimension scores. Center column: Inattention dimension scores. Right column: Combined scores (calculated as the sum of hyperactivity-impulsivity and inattention). Darker colored points indicate individuals with overlapping score data. 


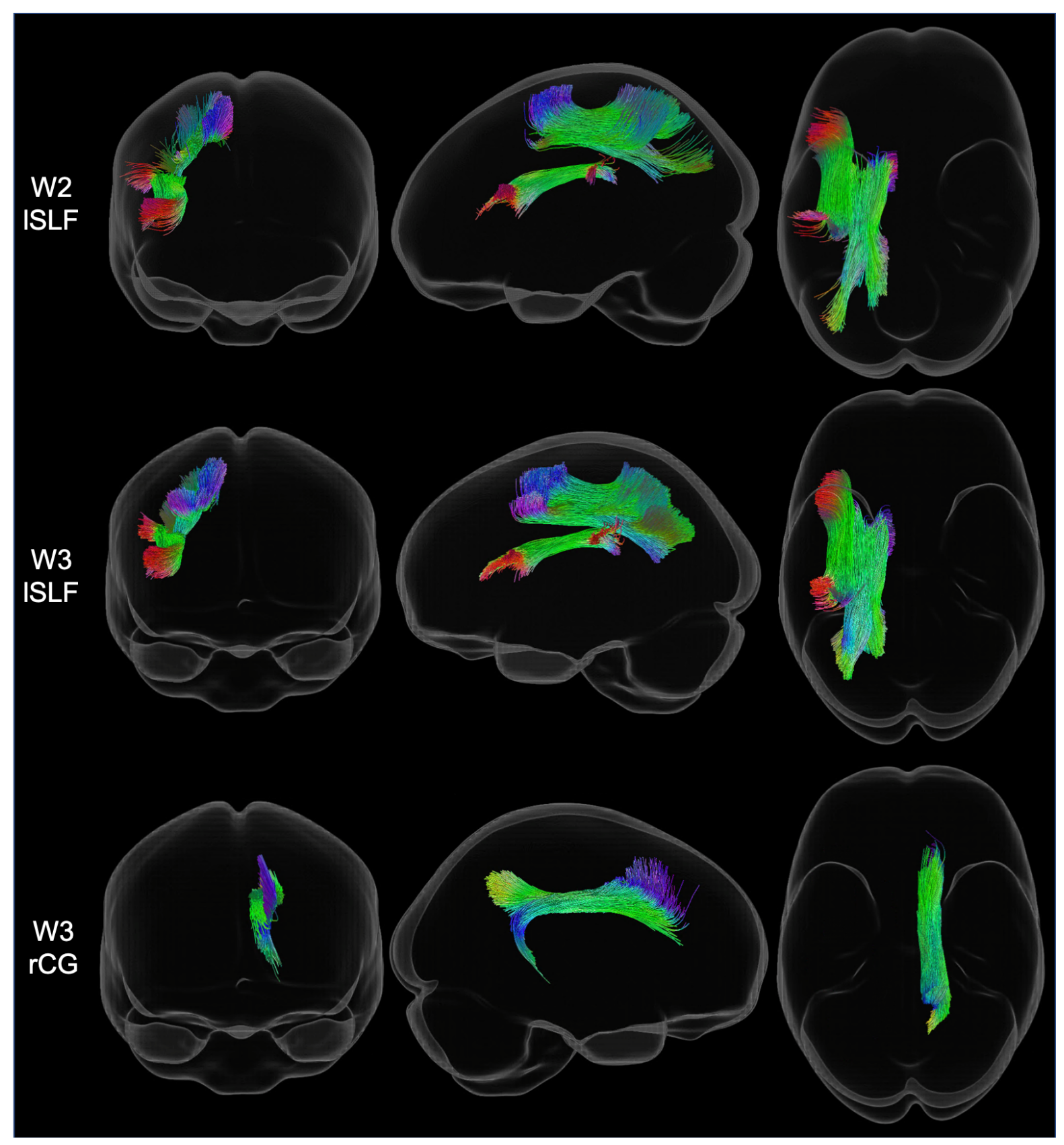

Figure S2. Tract-specific region-of-interest masks of the left superior longitudinal fasciculus (ISLF) at Wave 2 and Wave 3, and the right cingulum bundle (rCG) at Wave 3 colored by direction (red: leftright, green: anterior-posterior, blue: inferior-superior). Coronal (left column), sagittal (middle column), and axial (right column) views of the tract reconstructions from TractSeg (applied to the fiber orientation distribution templates) and displayed in glass brains for visualization. 
A

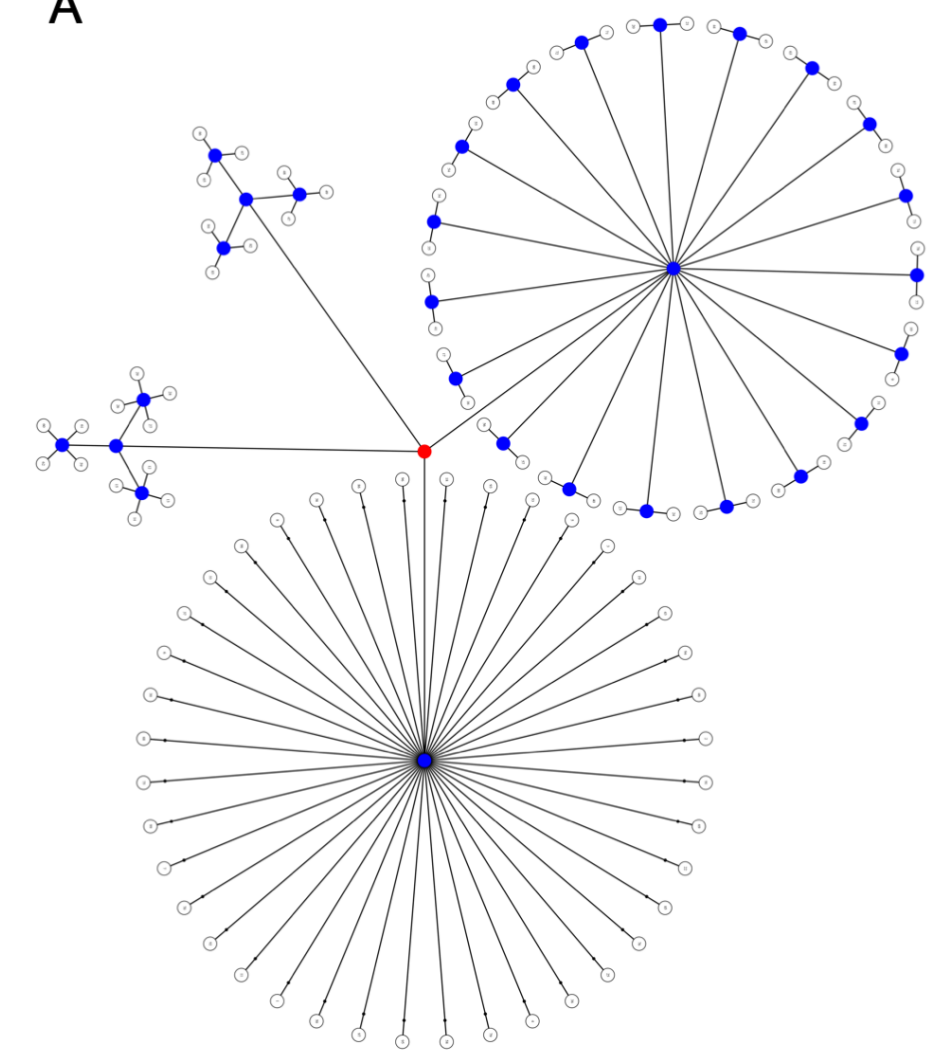

B

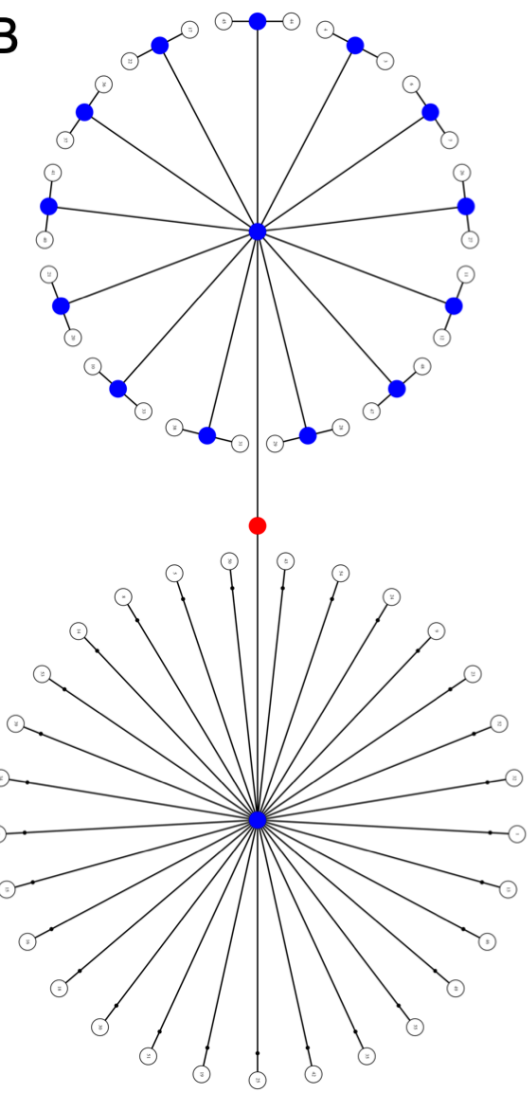

Figure S3. Visualization of the exchangeability block structure of (A) Wave 2 and (B) Wave 3, each represented in permutation trees. Each white dot is one individual and each group of white dots represents families of a specific size. At each permutation, branches beginning at blue dots can be permuted, while those beginning in red dots cannot. These permutation sets were generated with FSL PALM and used in connectivity-based fixel enhancement analysis to control for related siblings in each sample. 

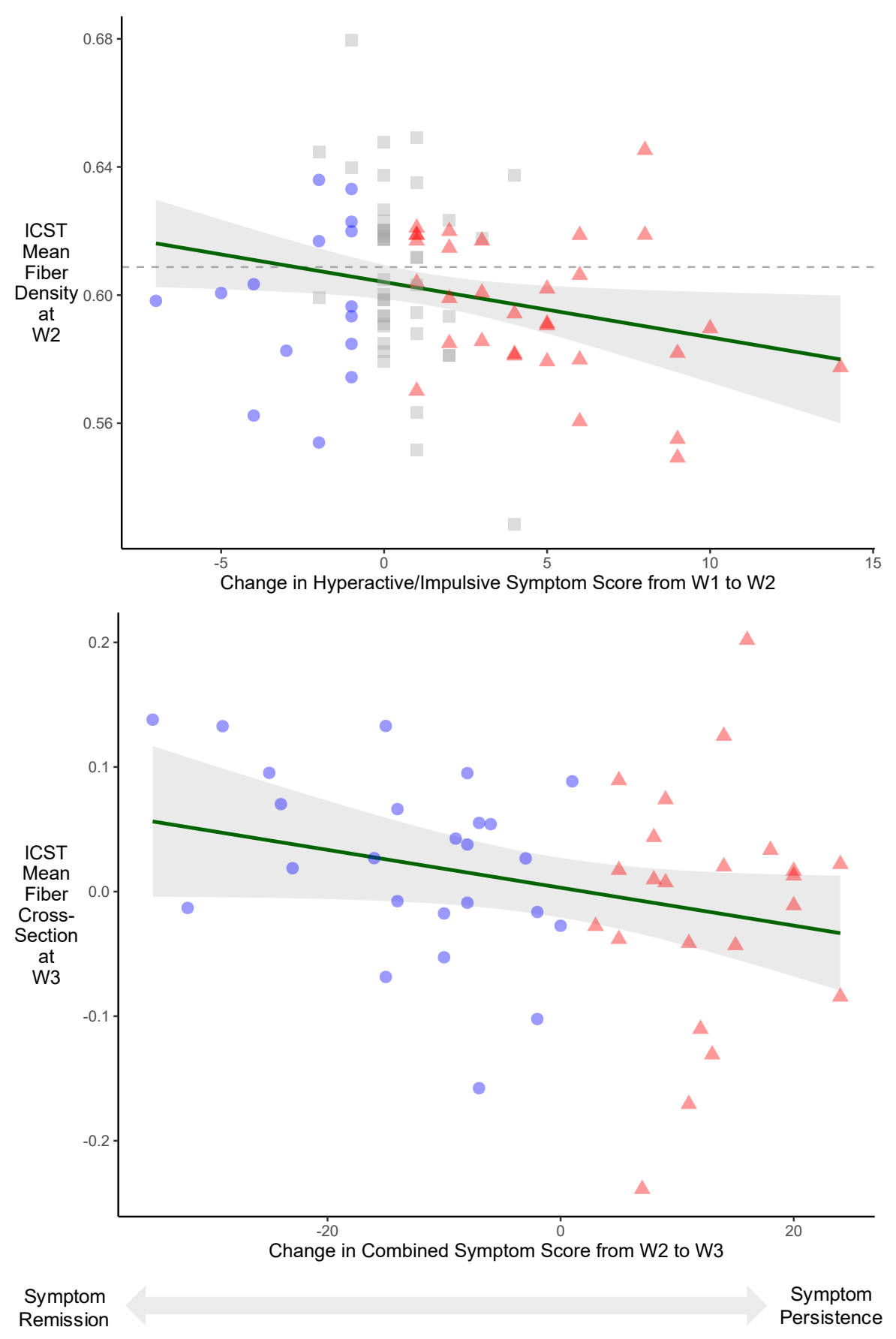

Figure S4. Graphs illustrating the relationship between left corticospinal tract (Top) mean fiber density values and change in hyperactive/impulsive symptom score from Wave 1 to Wave 2, and (Bottom) mean fiber cross-section values and change in combined symptom score from Wave 2 to Wave 3 . Both plots include solid green regression lines with $95 \%$ confidence intervals. For reference, the mean fiber density at Wave 2 for unaffected participants is marked by a dashed gray line. Change in symptom score was calculated as: $\Delta$ Score $=$ score follow-up - score baseline. Participants are shown as individual points, differentiated according to whether their change in symptom score was above or below the median change in score. 

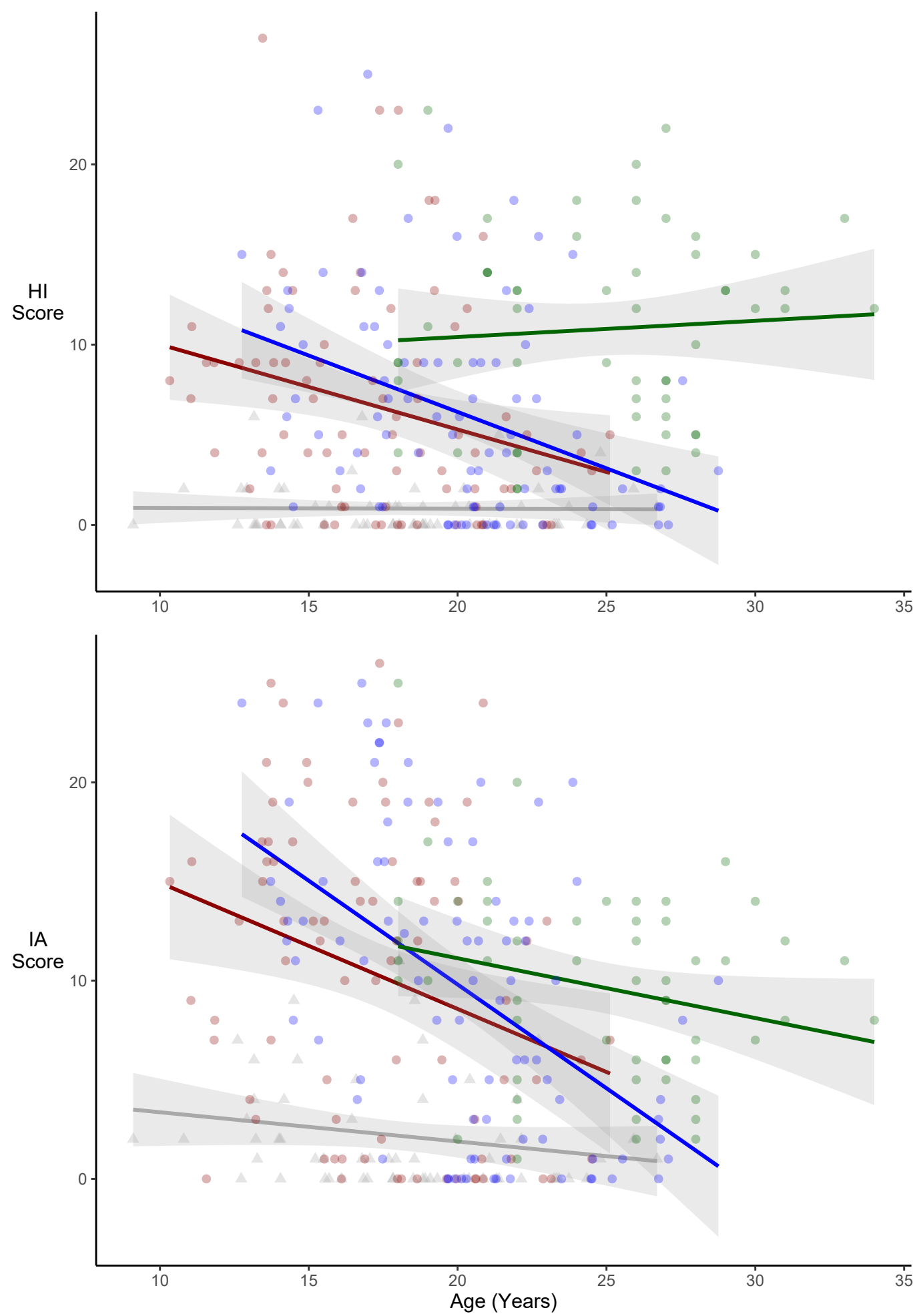

Figure S5. Participants from all waves pooled together (never affected are in gray triangles, and affected are circles colored according to wave in red [W1], blue [W2], and green [W3]) and plotted by age against symptom score in dimensions $\mathrm{HI}$ (Top) and IA (Bottom). Wave 3 included less participants and only those with a history of ADHD; scores were obtained with CAARS instead of CPRS. 